\title{
AREA OF BERNSTEIN-TYPE POLYNOMIALS
}

\author{
MARTIN E. PRICE
}

ABSTRACT. Bernstein polynomials in one variable are known to be total-variation diminishing when compared to the approximated function $f$. Here we consider the two variable case and give a counterexample to show they are not area-diminishing. Sufficient conditions are then given on a continuous function $f$ to insure convergence in area. A similar theorem is proved for Kantorovitch polynomials in the case $f$ is summable.

We consider the two-dimensional Bernstein polynomials $B_{n, m} f$, and the corresponding Kantorovitch polynomials $K_{n, m} f$, for functions $z=f(x, y)$ defined on the unit square $Q$. Sufficient conditions are gixen to insure the convergence in area of these polynomials. In particular if $f$ is summable and generalized absolutely continuous on $Q$, then $L K_{n, m} f \rightarrow \Phi f$ where $L$ is Lebesgue area, and $\Phi$ is the Cesari-Goffman generalized area; if $f$ is continuous and ACT, with $R$-integrable Tonelli lengths, then $L B_{n, m} f \rightarrow L f$.

For any $f$ defined on all of $Q$,

$$
B_{n, m} f(x, y)=\sum_{r=0}^{n} \sum_{s=0}^{m} f\left(\frac{r}{n}, \frac{s}{m}\right) p_{n, r}(x) p_{m, s}(y)
$$

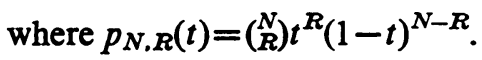

For summable $f$ on $Q$,

where

$$
K_{n . m} f(x, y)=\sum_{r=0}^{n} \sum_{s=0}^{m} I_{r, s} p_{n, r}(x) p_{m, s}(y)
$$

$$
I_{r, s}=(n+1)(m+1) \int_{r /(n+1)}^{(r+1) /(n+1)} \int_{s /(m+1)}^{(s+1) /(m+1)} f(\xi, \eta) d \xi d \eta
$$

If $f$ is continuous, $B_{n, m} f$ and $K_{n, m} f$ converge uniformly to $f$. Although the behavior of $B_{n, m} f$ for discontinuous functions is quite erratic,

Received by the editors March 20, 1971.

AMS (MOS) subject classifications (1970). Primary 26A63; Secondary 41A10.

Key words and phrases. Generalized area, generalized absolute continuity, Kantorovitch polynomials, Bernstein polynomials.

(c) American Mathematical Society 1974 
e.g. $\left[\mathbf{L}\right.$, p. 28], and $\left[\mathbf{P}_{1}\right]$, we have

Proposition 1. If $f$ is summable on $Q, K_{n, m} f$ converges in the $L_{1}$ sense to $f$.

Proof. For all $m, n, \int_{0}^{1} \int_{0}^{1} K_{n, m} f=\int_{0}^{1} \int_{0}^{1} f$ because $\int_{0}^{1} p_{N \cdot R}(t) d t=$ $1 /(N+1)$ for any $N$ and $R=0,1, \cdots, N$. Hence $\left\|K_{n, m} f\right\|_{1} \leqq\|f\|_{1}$. Choose a continuous $h$ such that $\|f-h\|_{1} \leqq \varepsilon / 3$. Then

$$
\begin{aligned}
\left\|f-K_{n, m} f\right\|_{1} & \leqq\|f-h\|_{1}+\left\|h-K_{n, m} h\right\|_{1}+\left\|K_{n, m} h-K_{n, m} f\right\|_{1} \\
& \leqq 2\|f-h\|_{1}+\left\|h-K_{n, m} h\right\|_{1} .
\end{aligned}
$$

Since $h$ is continuous, the last term is also at most $\varepsilon / 3$ for large $m$ and $n$, which completes the proof.

Cesari and later Goffman have defined equivalent areas for summable functions on $Q$. We give Goffman's version [ $\left.\mathbf{G}_{1}\right]$. Let

$$
\Phi f \equiv \inf _{\left\{p_{i}\right\}} \liminf _{i \rightarrow \infty} L\left(p_{i}\right)
$$

where $p_{i}$ are quasilinear functions converging $L_{1}$ to $f$ and the inf is tiken over all such sequences of $p_{i}$. $\Phi$ is lower semicontinuous with respect to $L_{1}$ convergence and coincides with $L$ for continuous $f$.

If $f(x, y)$ is continuous, the linear variation for fixed $y$ is denoted by $V_{x} f(y)$; similarly $V_{y} f(x)$. Their Lebesgue integrals, the Tonelli variations are $V_{x} f=\int_{0}^{1} V_{x} f(y) d y$ and $V_{y} f=\int_{0}^{1} V_{y} f(x) d x$. Correspondingly for summable $f(x, y)$, the linear generalized variations are $\varphi_{x} f(y)$ and $\varphi_{y} f(x)$ where variation in each case is computed only over points of linear approximate continuity. The generalized Tonelli variations are $\varphi_{x} f=$ $\int_{0}^{1} \varphi_{x}(y) d y$ and $\varphi_{y} f=\int_{0}^{1} \varphi_{y}(x) d x$. For continuous $f$ and $g$,

$$
L(f+g) \leqq L f+V_{x} g+V_{y} g
$$

and for summable $f$ and $g$,

$$
\Phi(f+g) \leqq \Phi f+\varphi_{x} g+\varphi_{y} g .
$$

A continuous $f(x, y)$ is ACT if $V_{x} f$ and $V_{y} f$ are finite and $f$ is absolutely continuous on almost all lines parallel to each coordinate axis. A summable $f$ is said to be $g$ ACT if $\varphi_{x} f$ and $\varphi_{y} f$ are finite, and there exists an $h \sim g$ such that $h$ is absolutely continuous on almost all lines parallel to each coordinate axis. Functions of gACT type may be "essentially discontinuous" i.e. every $h \sim f$ is nowhere continuous $\left[\mathbf{G}_{2}\right]$.

For finite valued $f(x)$ on $[0,1]$,

$$
B_{n} f(x) \equiv \sum_{r=0}^{n} f\left(\frac{r}{n}\right) p_{n, r}(x)
$$


and for summable $f$,

$$
K_{n} f(x) \equiv \sum_{r=0}^{n}(n+1)\left(\int_{r /(n+1)}^{(r+1) /(n+1)} f(\xi) d \xi\right) p_{n, r}(x)
$$

Let $V$ be total variation, $\varphi$ be variation over points of approximate continuity, $l$ the Jordan length, and $\lambda$ the length over points of approximate continuity. Then for all $n$,
(a) $V B_{n} f \leqq V f$,
(c) $l B_{n} f \leqq l f$,
(b) $V K_{n} f \leqq \varphi f$,
(d) $\lambda K_{n} f \leqq \lambda f$.

Part (a) is in [L]; (b) is in $\left[\mathbf{P}_{\mathbf{2}}\right]$; (c) and (d) follow from (a) and (b) by an integral-geometric formula of Cauchy and Steinhaus $\left[\mathbf{P}_{\mathbf{2}}\right]$. In virtue of the lower semicontinuity of $V$ and $l$ with respect to uniform convergence, and of $\varphi$ and $\lambda$ with respect to $L_{1}$ convergence, all four functionals converge as $n \rightarrow \infty$. It is thus reasonable to conjecture $L B_{n, m} f \rightarrow L f$ and $L K_{n, m} f \rightarrow \Phi f$ as $n, m \rightarrow \infty$ for appropriate classes of functions.

There is a major difference in the two variable case however. Construct a $C^{\infty}$ "rounded spike" function $f_{\varepsilon}$ on $Q$ which vanishes off a circular neighborhood $C_{\varepsilon}$ of $\left(\frac{1}{2}, \frac{1}{2}\right)$ and assumes the value 1 at $\left(\frac{1}{2}, \frac{1}{2}\right)$. By making the spike sufficiently thin, $L f_{\varepsilon}=1+\varepsilon$ for arbitrarily small positive $\varepsilon$. On the other hand $B_{22} f_{\varepsilon}=4 x y(1-x)(1-y)$ and is independent of the base radius $r_{\varepsilon}$ of the spike. Hence, though $f_{\varepsilon}$ is $C^{\infty}, L B_{22} f_{\varepsilon}>1+\varepsilon=L f_{\varepsilon}$ for some $\varepsilon$ in contrast to the relations (2). We now state the theorems.

THEOREM 1. If $f$ is $g A C T$, then $\lim _{n, m \rightarrow \infty} L K_{m . n} f=\Phi f$.

Proof. $\Phi$ is lower-semicontinuous with respect to $L_{1}$ convergence, so by Proposition $1, \lim \inf _{n, m \rightarrow \infty} L K_{n, m} f \geqq \Phi f$.

By (1b),

$$
\begin{aligned}
\Phi f & \leqq \lim \inf L K_{n, m} f \leqq \lim \sup L K_{n, m} f=\lim \sup \Phi K_{n, m} f \\
& \leqq \Phi f+\lim \sup \varphi_{x}\left(K_{n, m} f-f\right)+\lim \sup \varphi_{y}\left(K_{n, m} f-f\right) .
\end{aligned}
$$

It will be sufficient then to show (say) $\varphi_{x}\left(K_{n, m} f-f\right) \rightarrow 0$. Since $f$ is $g \mathrm{ACT}$, $\partial f / \partial x$ is summable, where $\partial f / \partial x$ is the partial derivative with sets of measure zero neglected in the difference quotient $\left[\mathbf{G}_{1}\right]$. Pick $h$ continuously differentiable on $Q$ such that $\|(\partial f / \partial x)-h\|_{1}<\varepsilon / 3$; i.e. $\varphi_{x}(f-H)<$ $\varepsilon / 3$ where $H(x, y)=\int_{0}^{x} h(t, y) d t$. Thus

$\varphi_{x}\left(K_{n, m} f-f\right) \leqq \varphi_{x}(f-H)+V_{x}\left(H-K_{n, m} H\right)+V_{x}\left(K_{n, m} H-K_{n, m} f\right)$.

The first term is $<\varepsilon / 3$, and so is the second for large $n$ and $m$ because $\left(\partial K_{n, m} H / \partial x\right) \rightarrow(\partial H / \partial x)$, since $H$ is $C^{1}$. The proof of this follows from showing $\left|\left(\partial K_{n, m} / \partial x\right)-\left(\partial B_{n, m} / \partial x\right)\right|$ to be small, and then using the corresponding result for $B_{n, m}$ which is proved in [B]. 
For the third term, we need a lemma which holds for any summable function.

LEMMA. For $F(x, y)$ summable on $Q$ and all $m$ and $n, V_{x} K_{n, m} F \leqq \varphi_{x} F$ (and $V_{y} K_{n, m} F \leqq \varphi_{y} F$ ).

Proof.

$$
\begin{aligned}
V_{x} K_{n, m} F & =\int_{0}^{1} \int_{0}^{1}\left|\frac{\partial K_{n, m} F}{\partial x}\right| d x d y \\
& =n \int_{0}^{1} \int_{0}^{1}\left|\sum_{s=0}^{m} \sum_{r=0}^{n-1}\left(I_{r+1, s}-I_{r, s}\right) p_{n-1, r}(x) p_{m, s}(y)\right| d x d y \\
& \leqq n \sum_{r=0}^{n-1} \sum_{s=0}^{m} \int_{0}^{1} \int_{0}^{1}\left|I_{r+1, s}-I_{r, s}\right| p_{n-1, r}(x) p_{m, s}(y) d x d y \\
& =\frac{1}{m+1} \sum_{r=0}^{n-1} \sum_{s=0}^{m}\left|I_{r+1, s}-I_{r, s}\right| .
\end{aligned}
$$

But

$$
\begin{aligned}
\left|I_{r+1, s}-I_{r, s}\right| \leqq(m+1) & \int_{s /(m+1)}^{(s+1) /(m+1)}(n+1) \\
& \cdot\left|\int_{(r+1) /(n+1)}^{(r+2) /(n+1)} F(\xi, \eta) d \xi-\int_{r /(n+1)}^{(r+1) /(n+1)} F(\xi, \eta) d \xi\right| d \eta
\end{aligned}
$$

and so

$$
\begin{aligned}
V_{x} K_{n, m} F \leqq \int_{0}^{1}(n+1) \sum_{r=0}^{n-1} \mid \int_{(r+1) /(n+1)}^{(r+2) /(n+1)} F(\xi, \eta) d \xi & \\
& -\int_{r /(n+1)}^{(r+1) /(n+1)} F(\xi, \eta) d \xi \mid d \eta
\end{aligned}
$$

For almost all $\eta \in[0,1], F(\xi, \eta)$ is a summable function of $\xi$. For these $\eta$, the expression inside the first integral is at most $\varphi_{x} F(\eta)$. The proof is essentially that of (2)(b). Thus the right hand side of (3) is at most $\int_{0}^{1} \varphi_{x} F(\eta) d \eta=\varphi_{x} F$ which completes the proof.

Now let $F=H-f . F$ is summable, and so by the lemma

$$
V_{x}\left(K_{n, m} H-K_{n, m} f\right)=V_{x}\left(K_{n, m}(H-f)\right) \leqq \varphi_{x}(H-f)<\varepsilon / 3 .
$$

Hence $\varphi_{x}\left(K_{n, m} f-f\right)<\varepsilon$ for large $n$ and $m$ which completes the proof of Theorem 1.

For the next theorem, set $l_{x} f=\int_{0}^{1} l_{x} f(y) d y$ where $l_{x} f(y)$ is the Jordan length in the $x$-direction of a section at $y$. Similarly define $l_{y} f$.

THEOREM 2. If $f$ is $A C T$ and $l_{x} f$ and $l_{y} f$ are $R$-integrable, then $\lim _{n, m \rightarrow \infty} L B_{n, m} f=L f$. 
Proof. Since $B_{n, m} f \rightarrow f$ uniformly, $\lim \inf _{n, m \rightarrow \infty} L B_{n, m} f \geqq L f$. By (1a), it is sufficient to show as in Theorem 1 , that (say) $V_{x}\left(\bar{B}_{n, m} f-f\right) \rightarrow 0$. Let $h$ and $H$ be as in Theorem 1 with $V_{x}(f-H)<\varepsilon / 4$. Then

$$
V_{x}\left(B_{n, m} f-f\right) \leqq V_{x}(f-H)+V_{x}\left(H-B_{n, m} H\right)+V_{x}\left(B_{n, m}(H-f)\right) .
$$

The first term is at most $\varepsilon / 4$, as is the second for large $n$ and $m$, because $\left(\partial B_{n, m} H / \partial x\right) \rightarrow(\partial H / \partial x)$ uniformly [B]. For the third term, it is necessary to show $V_{x}(f-H)(y)$ is $R$-integrable.

Since $l_{x} f$ is $R$-integrable, $l_{x} f(y)$ and hence $V_{x} f(y)$ is bounded for $y \in$ $[0,1]$. Since $H$ is $C^{1}, V_{x}(f-H)(y)$ is bounded. In addition, $V_{x}(f-H)(y)$ is continuous almost everywhere. To see this, pick $y_{0}$ from the full measure set where simultaneously $f\left(x, y_{0}\right)$ is absolutely continuous as a function of $x$, and $l_{x} f(y)$ is continuous as a function of $y$. Consider a sequence $y_{n} \rightarrow y_{0}$, and correspondingly the $l_{x} f\left(y_{n}\right)$ and $l_{x} H\left(y_{n}\right)$. Since $H$ is $C^{1}$, $H\left(x, y_{0}\right)$ is an absolutely continuous function of $x$. By theorems in [A-L], $l_{x}(f-H)\left(y_{n}\right) \rightarrow l_{x}(f-H)\left(y_{0}\right)$ which implies $V_{x}(f-H)\left(y_{n}\right) \rightarrow V_{x}(f-H)\left(y_{0}\right)$. Thus $V_{x}(f-H)(y)$ is continuous at almost all $y$ and is $R$-integrable.

For arbitrary $F(x, y)$, a computation similar to the lemma shows

for all $n, m$. Thus

$$
V_{x} B_{n, m} F \leqq \frac{1}{m+1} \sum_{s=0}^{m} V_{x} F\left(\frac{s}{m}\right)
$$

$$
V_{x} B_{n, m}(H-f) \leqq \frac{1}{m+1} \sum_{s=0}^{m} V_{x}(H-f)\left(\frac{s}{m}\right)
$$

which converges to $V_{x}(H-f)$ by $R$-integrability of $V_{x}(H-f)(y)$. Hence for large $m$ and all $n$, the right hand side of (4) is less than $2(\varepsilon / 4)=\varepsilon / 2$. For the same $m$ and $n$,

$$
V_{x}\left(B_{n, m} f-f\right) \leqq \frac{\varepsilon}{4}+\frac{\varepsilon}{4}+\frac{\varepsilon}{2}=\varepsilon,
$$

and the same computation for $y$ shows $V_{y}\left(B_{n, m} f-f\right) \rightarrow 0$. Therefore $\lim \sup L B_{n, m} f \leqq L f$ which completes the proof.

\section{REFERENCES}

[A-L] C. R. Adams and H. Lewy, On convergence in length, Duke Math. J. 2 (1936), 19-26.

[B] P. Butzer, On two dimensional Bernstein polynomials, Canad. J. Math. 5 (1953), 107-113. MR 14, 641.

[G $\mathbf{G}_{1}$ C. Goffman, Lower semi-continuity and area functionals. I. The non-parametric case, Rend. Circ. Mat. Palermo (2) 2 (1953), 203-235. (1954). MR 16, 457.

$\left[\mathbf{G}_{2}\right] \longrightarrow$, Two remarks on linearly continuous functions, J. Math. Mech. 16 (1967), 1227-1228. MR 37 \#4214. 
[L] G. Lorentz, Bernstein polynomials, Mathematical Expositions, no. 8, Univ. of Toronto Press, Toronto, 1953. MR 15, 217.

$\left[\mathbf{P}_{1}\right] \mathrm{M}$. Price, On the variation of the Bernstein polynomials of a function of unbounded variation, Pacific J. Math. 27 (1968), 119-122. MR 38 \#1441.

$\left[\mathbf{P}_{2}\right]-$ Kantorovitch polynomials diminish generalized length, Canad. Math. Bull. 15 (1972).

Department of Mathematics, Wayne State University, Detrort, Michigan 48202

Current address: Department of Mathematics, Framingham State College, Framingham, Massachusetts 01701 